ratios $1: 10: 100$. More significantly, the stress required for failure after a given number of cycles increased in the same ratios $(\sim 1: 1.5: 2)$ as the static ultimate tensile strength. Work at liquid helium temperature, now proceeding, shows still further increase in fatigue life, although the correlation with the change in ultimate tensile strength is no longer valid. No one seemed to have a ready explanation for these rather surprising results.

The afternoon session of the meeting was in the form of a general discussion, in which a panel of members of the Metal Physics Committee attempted to answer questions suggested by the local section. The current interest in fatigue problems was shown by the question, "Why have very high-strength aluminium alloys a fatigue strength no better than that of medium-strength alloys ?", which produced, in effect, a continuation of the morning discussion.

J. W. CHRISTIAN

\section{AROMATIC SUBSTITUTION}

$\mathrm{O}^{\mathrm{A}}$ March 29 a symposium was held by the Chemistry Department of Queen Mary College, London, on "Aromatic Substitution", with Prof. E. R. H. Jones in the chair. The six contributions covered various aspects of electrophilic, nucleophilic and homolytic substitution.

Prof. M. J. S. Dewar reviewed the general theory of aromatic substitution in terms of the Wheland transition-state treatment and the molecular orbital method. The latter method may be used to calculate the position of substitution in polycyclic alternant hydrocarbons, and the results obtained are generally in agreement with the experimental results. The theory does not take into account steric hindrance, which appears to occur particularly in $\alpha$-naphthalenic positions such as the 4-position in phenanthrene. Electrophilic substitution in heterocyclic systems was then discussed and the molecular orbital theory shown to be applicable in this field. Finally, Prof. Dewar described work being carried out on the nitration of quinoline which shows that this reaction proceeds by different mechanisms in acetic anhydride and sulphuric acid solution. In the subsequent discussion Prof. E. D. Hughes emphasized the importance of the reagent and also the effect of temperature on the orientation of the substituent. Prof. Dewar agreed and illustrated the effect of the reactivity of the reagent.

Dr. G. Baddeley ${ }^{1}$ discussed the Friedel-Crafts acylation and alkylation of aromatic compounds, and emphasized the effect of the reactivity of the reagent on the nature of the product. With aluminium chloride in methylene chloride solution the usual orientation rules were observed, whereas if the solvent was nitromethane or ether the reagent was less reactive and 'abnormal' products were obtained. This was illustrated with numerous examples such as the substitution of anthracene, which occurs in the 9 -position in methylene chloride solution but in the 1- and 2-positions in nitromethane solution. Dr. Baddeley ascribed the abnormal orientation to complex formation between the catalyst and solvent, attack by the bulky complex being liable to steric hindrance. Dr. E. A. Braude suggested that reversibility of the reaction might account for the abnormal orientation in polar solvents, but Dr. Baddeley said that, if excess aluminium chloride is avoided and the hydrogen chloride removed, the reactions are not reversible.

Dr. P. B. D. de la Mare then read a paper on the chlorination and bromination of aromatic compounds. He stated that these reactions, when carried out by a solution of the halogen in hydroxylic solvents, can be shown kinetically to involve molecular halogen, but that brominations by aqueous hypobromous acid have a kinetic form which implies that the electrophilic reagent carries a full positive charge. Dr. de la Mare then compared molecular halogenation with ionic nitration and showed that the results suggest that, while the positively charged reagent evolkes the inductive effect of the substituent most powerfully, the molecular reagent evokes the conjugative electromeric effect more powerfully.

The afternoon session was opened by Dr. N. B. Chapman, who gave a short survey of nucleophilic substitution and then proceeded to discuss the nucleophilic displacement of halogen. He directed attention to possible duality of mechanism (analogy with saturated systems) and to the possibility of complexity of mechanism in bimolecular processes, and outlined the structural requirements for nucleo. philic substitution in homocyclic aromatic compounds. Dr. Chapman then discussed the quantummechanical background to the nucleophilic displacement of halogen in heterocyclic compounds (monoand di-aza-naphthalenes) and gave an account of some kinetic investigations which have provided kinetic results for comparison with the approximate quantum-mechanical calculations; the agreement between the calculated and observed activation energies is not very good. In the discussion Dr. E. Parker suggested that the structure of the transitionstate might involve $d$-orbitals, and this suggestion was generally accepted as being probable. Prof. Dewar suggested that a comparison of calculated activation energies with observed rates of reaction might be more correct and lead to a better agreement between theory and experiment.

Prof. W. Bradley discussed nucleophilic substitutions in which hydrogen is replaced by an anion, the formation of $o$-nitrophenol from nitrobenzene and potassium hydroxide being a typical example. The reactivity of the nucleus towards nucleophilic substitution decreases from anthracene to naphthalene to benzene, and nucleophilic substitution is facilitated by a carbonyl or nitro group attached to the nucleus, the positions ortho-para to the group being preferentially attached. Numerous examples show that, on direct hydroxylation with potassium hydroxide, mono-ketones and di-ketones yield respectively mono- and di-hydroxy compounds, and substitution by other anions follows a similar course. Prof. Bradley then gave several examples where substitution by an anion is accompanied by self-union of two molecules of the aromatic reactant, usually via a carbon atom para to a carbonyl group. He expressed the view that this is due to the anion functioning as a base and removing a proton from the aromatic reactant.

Prof. D. H. Hey opened his paper on homolytic substitution ${ }^{2}$ by outlining the reactions by which phenyl radicals may be generated, and he then briefly discussed the partial rate factors for the phenylation of a series of mono-substituted benzene derivatives. He described the phenylation of toluene, ethylbenzene isopropylbenzene and tertbutylbenzene; with the first three of these compounds the reactions are complicated by dimerization due to side-chain attack. 
In the case of tertbutylbenzene this does not occur, but there is a marked steric opposition to substitution at the ortho positions which results in the first example of overall deactivation encountered in homolytic substitution. Prof. Hey then outlined some recent investigations on the proportions of the isomeric phenylpyridines formed in the phenylation of pyridine using a number of different sources of phenyl radicals. The results were independent of the source of the radical, except when the source was phenylazotriphenylmethane. This anomalous result was shown to be due to the formation of a by-product in which it appeared that the triphenylmethyl grouping was incorporated.

In view of the interest aroused by this symposium and by one held last year ${ }^{3}$ at Queen Mary College, it has been decided to make the symposium an annual event, dealing with some topic of current interest in the field of organic reaction mechanisms.

M. F. ANSELL

M. J. S. DEWAR

I Baddeley, G., Quart. Rev., 8, 355 (1954).

${ }^{2}$ Hey, D. H., et al., J. Chem. Soc., 3352 (1954), and earlier papers.

${ }^{3}$ Ansell, M. F., and Dewar, M. J. S., Nature, 173, 898 (1954).

\section{SOIL ZOOLOGY}

\section{EASTER SCHOOL AT NOTTINGHAM}

$\mathrm{T}$ HE second Easter school in agricultural science in the University of Nottingham School of Agriculture, Sutton Bonington, held during April 1-7, was devoted to soil zoology. This symposium and colloquium were limited to a membership of seventy, which included not only a large number of specialists in soil zoology and pedology, among whom were several distinguished scientific workers from other European countries, but also a number of biologists who wished to acquaint themselves with this field. The function of the meeting, therefore, was to provide a tilting-ground for discussion of papers delivered by specialists and, in addition, tuition by lecture, demonstration and practical class-work for those less familiar with the ramifications of this rapidly growing subject.

After the address of welcome by Prof. E. G. Hallsworth, the opening paper, on soil animals, by Prof. W. Kühnelt (Vienna), and the subsequent paper by Dr. P. W. Murphy (Rothamsted) on the ecology of the fauna of the forest soils, served as introductions to soil zoology and enabled the later more specialized contributions to be viewed in their ecological perspective. The difficulty of extracting animals from the soil in a condition which permits identification and in quantities which reflect the sizes of field populations has long been recognized. The paper by Mr. A. Macfadyen (Oxford), on a comparison of sampling methods for soil arthropods, was therefore welcomed by the entomologists present. An informal address on flotation methods of extraction was also given by Dr. F. S. J. Hollick (Cambridge). Dr. F. Raw's paper on a flotation extraction process for soil micro-arthropods was dernonstrated in his absence by his colleagues, Mr. J. M. Dobson and Mr. J. W. Stephenson (Rothamsted). As a direct result of careful extraction methods, information on the distribution of animals in the soil is increasing. This was well shown by the studies of Mr. D. H. Murphy (Durham) on long-term changes in Collembola populations, with special reference to the fauna of moorland soils; by Mr. N. Haarløv (Copenhagen) in his contribution on vertical distribution of mites and Collembola in relation to soil structure; and by Dr. C. Overgaard Nielsen (Femmøller, Denmark) in his survey of a year's results obtained by a recent method for the extraction of soil-inhabiting enchytraeid worms. The roles and distribution of earthworms were dealt with in two papers: Dr. W. J. Guild (Edinburgh) on earthworms and soil structure, and Dr. J. E. Satchell (Nature Conservancy, Grangeover-Sands) on some aspects of earthworm ecology.

Although most of the practical difficulties in the identification of soil animals were adequately explained in the practical classes, Dr. G. O. Evans (British Museum) gave a most useful talk on the problems encountered in naming terrestrial mites. The general biology of some of the less-worked groups was covered by Dr. B. G. Peters (Rothamsted), speaking on soil-inhabiting nematodes; by Mr. J. G. Blower (Manchester), on myriapods as soil animals; and by Mr. W. V. Harris (Commonwealth Institute, London-in absentia), on termites and the soil, this last lecture being introduced by Mr. W. A. Sands (Commonwealth Institute). A group with which the average zoologist is even less familiar, that of the predaceous fungi, was very ably dealt with by Dr. C. L. Duddington (London) and Dr. Mary Peach (London).

The relations of soil type and agricultural practice to the fauna of agricultural land were outlined in two lectures by Prof. W. Tischler (Kiel). The penultimate session was almost entirely devoted to the more practical aspects of soil ecology. A brief review was given by Mr. E. B. Brown (National Agricultural Advisory Service, Cambridge) in his paper on some current British soil pest problems. Mr. F. G. W. Jones (Cambridge) gave particular attention to one class of pests in his paper on quantitative methods for the estimation of cyst-forming eolworms in the soil. Some of the interesting aspects of the application of insecticides to soils were discussed by $\mathrm{Mr}$. J. G. Sheals (Glasgow) in the effects of DDT and $\mathrm{BHC}$ on soil Collembola and Acarina.

On the last morning Prof. W. L. Kubiëna (Madrid) showed a series of coloured slides illustrating the micro-structure of soil, paying special attention to the humus type. This was a fitting end to the meeting, as the cardinal importance of animal activity in the determination of humus type was demonstrated. Moreover, Prof. Kubiëna's remarks helped pedologists and zoologists alike to a more rigorous appreciation of the terms 'mull', 'mor' and 'moder', terms often used and as often misused during the meeting. On the last morning also, a number of valuable contributions which it had unfortunately been found impossible to incorporate in the formal proceedings were read 'by title', their texts having been circulated during the course and opportunity given for their discussion.

Several hours each day were devoted to practical demonstrations of various techniques, with adequate opportunities for testing them individually. All the demonstrations attracted considerable attention, and though it is perhaps invidious to single out any particular one, special interest was shown in the soilsectioning technique developed by Mr. N. Haarløv and Dr. T. Weis-Fogh.

In general, this meeting at Sutton Bonington was a great success, and much of the credit is due to the exceptional facilities provided at the School of 